\title{
Scale-free energy dissipation and dynamic phase transition in stochastic sandpiles
}

\author{
Bosiljka Tadić* \\ Jožef Stefan Institute, P.O. Box 3000, 1001 Ljubljana, Slovenia
}

\begin{abstract}
We study numerically scaling properties of the distribution of cumulative energy dissipated in an avalanche and the dynamic phase transition in a stochastic directed cellular automaton [B. Tadić and D. Dhar, Phys. Rev. Lett. 79, 1519 (1997)] in $d=1+1$ dimensions. In the critical steady state occurring for the probability of toppling $p \geq p^{\star}=0.70548$, the dissipated energy distribution exhibits scaling behavior with new scaling exponents $\tau_{E}$ and $D_{E}$ for slope and cut-off energy, respectively, indicating that the sandpile surface is a fractal. In contrast to avalanche exponents, the energy exponents appear to be $p$-dependent in the region $p^{\star} \leq p<1$, however the product $\left(\tau_{E}-1\right) D_{E}$ remains universal. We estimate the roughness exponent of the transverse section of the pile as $\chi=0.44 \pm 0.04$. Critical exponents characterizing the dynamic phase transition at $p^{\star}$ are obtained by direct simulation and scaling analysis of the survival probability distribution and the average outflow current. The transition belongs to a new universality class with the critical exponents $\nu_{\|}=\gamma=1.22 \pm 0.02, \beta=0.56 \pm 0.02$ and $\nu_{\perp}=0.761 \pm 0.029$, with apparent violation of hyperscaling. Generalized hyperscaling relation leads to $\beta+\beta^{\prime}=(d-1) \nu_{\perp}$, where $\beta^{\prime}=0.195 \pm 0.012$ is the exponent governed by the ultimate survival probability.
\end{abstract}

\section{INTRODUCTION}

Open driven systems exhibiting self-organized critical states [1] are usually modeled by sandpile-type automata, in which sand grains are added slowly to the system and its evolution is monitored in terms of collective sandslides (avalanches). With additional complexity due to stochastic character of the relaxation rules [2 [5], stochastic cellular automata models have proven useful in understanding certain aspects of granular flow [3] 5] in realistic granular materials and in stochastic biological processes [6]. On the other hand, sandpile automata models are interesting from the theoretical point of view since both role of the dynamic conservation law - conservation of the number of grains - and emergent spatial structures can be easily monitored.

Recently a model with probabilistic toppling and directed mass flow has been proposed [7], in which the probability of toppling $p$ represents a control parameter originating either from random variations of sticking properties between grains, or from stochastic external conditions (wetting and drying properties). Another realization is related to stochastic processes in biological systems such as random dispersion of particles, which are added from the outside and evacuated from the system only when its response lasts longer than a fixed time $T_{0}$, measured on the internal time scale of the process. Particles are transferred among communicating cells according to probabilistic rules, however, for response times shorter than $T_{0}$ they are held inside the system. Therefore, each cell contains a certain number of particles, which varies with time. The probabilistic character of the particle transfer between connected cells can be attributed to mechanisms which depend on general condition of the system. We consider the case $T_{0} \equiv L$, where $L$ is the linear system size.

The relaxation rules of the model in $d=1+1$ dimensions are: if $h(i, j) \geq h_{c}=2$ then with the probability $p$ the relaxation occurs as follows:

$$
h(i, j) \rightarrow h(i, j)-2 ; h\left(i+1, j_{ \pm}\right) \rightarrow h\left(i+1, j_{ \pm}\right)+1 .
$$

Here $h(i, j)$ is the dynamic variable, i.e., the height (number of particles) at site $(i, j)$, and $\left(i+1, j_{ \pm}\right)$are neighboring downstream sites on a 2-dimensional square lattice oriented downwards. Due to directed mass transport the dynamics of this model is anisotropic leading to selfaffine relaxation clusters in $d=2$ (see Fig. 1). Thus the model can also be viewed as directional lines in $d=1+1$ dimensions, in which the instability can propagate in one spatial dimension back and forth, whereas the temporal dimension is strictly directed. The system is driven by adding particles from the outside, one at a time at a random position along the first (top) row. Only sites which are connected to toppled sites at the previous time step are considered as candidates for toppling. Perturbation can then be transferred from an active (toppled) site to two forward neighbors. Periodic transverse boundaries are assumed and all candidate sites are updated in parallel. The probabilistic character of the relaxation rules produces a ragged structure of heights. [A transverse section through the pile is shown in Fig. 1 (top)]. Two top particles from the surface $h(i, j)$ are taken away from the system when the active site is on the lower boundary, i.e., $i=L$.

It should be stressed that according to the above relaxation rules transport of grains is independent of the 
relative heights of neighboring sites. Therefore, it may occur that at some sites height difference in the direction of transport is negative, and thus the system performs work in order to maintain the transport. A nice example of this dynamic rules was found recently in biological transport processes which are mediated by so called molecular motors [8]. These are protein molecules that can use excess energy from the chemical reactions in the fuel (adenosine triphosphate) and perform mechanical work.

It has been understood [7] that the automaton exhibits self-organized criticality for the range of values of the control parameter $p \geq p^{\star}$, where $p^{\star}=p_{c}^{S D P}=0.7054853(5)$ is the percolation threshold for the site directed percolation [9] on the square lattice. Due to the dynamic conservation law - conserved number of grains in the interior of the pile - and the probabilistic relaxation rules which are locally like the generalized site-bond directed percolation (see below), the system exhibits emergent spatial structure, as discussed in detail in Ref. [7]. Moreover, the avalanche exponents for the integrated probability distributions of duration $P(T) \sim T^{-\left(\tau_{t}-1\right)}$ and size of avalanches, $D(s) \sim s^{-\left(\tau_{s}-1\right)}$, are expressible in terms of the standard directed percolation exponents in $d=1+1$ dimensions as follows [7]:

$$
\tau_{t}-1 \equiv \alpha=(d-1) \zeta_{D P}-\left(\beta / \nu_{\|}\right)_{D P},
$$

and $\tau_{s}=2-1 / \tau_{t}$, and the anisotropy exponent $\zeta=\zeta_{D P}$. Subscript $D P$ refers to directed percolation, and $\beta$ and $\nu_{\|}$are the critical exponents for the order parameter and parallel correlation length, respectively.

In the present work we extend the study of the model of Ref. [7] in two ways: (1) We study the probability distribution of the potential energy dissipated in a relaxation event (avalanche) due to grains drop from higher to lower positions at the fluctuating sandpile surface. This distribution is unique for the dynamic sandpile models with ragged spatial structure and has no counterpart in the directed percolation processes. Thus we expect that the exponents characterizing its scaling properties are new. (2) We analyze the behavior of the system close to the phase transition point in terms of $(i)$ scaling properties of the survival probability distribution for $p<p^{\star}$ and (ii) by determining the time averaged outflow current $<J(p)>$, which behaves as an order parameter. The outflow current results from avalanches which last longer than the system size $T \geq L$. The average is taken over $e x$ ternal time scale, which is measured in number of added grains. The internal current at time $T<L$ is defined as $<j(T, p)>\sim T^{-\alpha} m(T, p)$, where $m(T, p)$ is the average flux of particles at time $T$, and $T^{-\alpha}$ is the probability that an avalanche survives $T$ steps. In the steady state outflow current $\langle J(p)\rangle$ balances the input current, which is one particle per time step, and thus it is equal to one. For $p<p^{\star}$ the system ceases to conduct parti- cles, and the outflow current drops to zero for $\ell \gg \xi(p)$ and finite lattice size $L$ as

$$
<J(p)>\sim(\delta p)^{\beta} g(L / \xi(p)),
$$

where $\delta p \equiv\left(p-p^{\star}\right) / p^{\star}$ measures the distance from the steady state and $\xi(p) \sim(\delta p)^{-\nu_{\|}}$is the parallel correlation length. The set of critical exponents is determined by the appropriate scaling fits and using the scaling relations that are valid in the present dynamical model (see Sections IV and V).

The organization of the paper is as follows: In Sec. II the phase diagram of the system is calculated numerically for finite lattice size $L$. In Sec. III we study scaling properties of the distribution of energy dissipated in avalanches. In Sec. IV we present detailed numerical analysis of the phase transition. Summary of the universal scaling exponents and the discussion of the results is given in Sec. V.

\section{PHASE DIAGRAM}

Due to probabilistic dynamic rules the avalanches in this model show a fractal structure; an example is shown in Fig. 1 (bottom). In the limit $p=1$ this model reduces to the deterministic directed model with compact avalanches, which has been introduced and solved exactly in Ref. [10].

As discussed in detail in Ref. [7], for $p^{\star} \leq p<1$ the relaxation rules at each site of the system may be visualized as the rules of a Domany-Kinzel cellular automaton 11] of generalized site-bond directed percolation (DP), with probabilities $P_{1}$ and $P_{2}$ that a toppling occurs if one or two particles, respectively, drop at that site. According to Eq. (1), we have $P_{1} \equiv p \rho$, where $\rho$ is the probability that the site has height $h \geq 1$, and $P_{2} \equiv p$ by definition. In contrast to DP, in the present dynamic model the state is being systematically built up after each avalanche, and the probability $\rho$ was found to vary with the distance $\ell$ from the top of pile as [7] $\rho(\ell, p)=\rho^{\star}(p)-A(p) \ell^{-x}$. Here $x=1 / \nu_{\|}^{D P}$ (see inset to Fig. 2) is the inverse parallel correlation length exponent of the directed percolation [12]. In the above formula $\rho^{\star}(p)$ are the values of $\rho(\ell, p)$ reached at $\ell \rightarrow \infty$. Notice that the distance $\ell$ from the top of the pile is equivalent to the duration $T$ of avalanches. In Fig. 2 we show time-averaged $\left\langle\rho^{\star}(p)>\right.$ vs. $p$ obtained numerically and averaged over lower third of the lattice with $L=100$, for various values of $p$. Two types of initial conditions are used: (a) full lattice (all lattice sites are occupied by at least one particle), and (b) half-full lattice (half of the sites which are selected randomly have zero heights and the rest of sites are occupied). In both cases, for the initial set of probabilities $\left(p, \rho^{\star}\right)$ in the region to the right of $p^{\star}$ the system selforganizes (after some transient time) to sitting close to the DP critical line (cf. Fig. 2). Left of the line $p=p^{\star}$ 
different initial conditions lead to separate final states. We used $8 \times 10^{6}$ time steps for each point. Notice that due to finite size of the lattice $\left\langle\rho^{\star}\left(p^{\star}\right)>\right.$ is still somewhat smaller than one (indicated by dotted line in Fig. 2 ), and that a dynamical hysteresis occurs in the region $0.5 \leq p<p^{\star} 13$. At $p^{\star}$ an instability-building up of heights - starts at lower boundary of the pile and proliferates inside, reaching the first row for probability of toppling exactly $p=1 / 2$. Apart from the finite-size effects, the phase diagram in Fig. 2 is in agreement with general theoretical considerations given in Ref. [7].

\section{DISSIPATED ENERGY DISTRIBUTION}

Due to the probabilistic character of the dynamic relaxation rules in Eq. (11) for $p<1$ and the conservation of particles in the interior of the pile, our dynamic model exhibits the emergent spatial structure [7], which is characterized by rough surface $h(i, j)$ embeded in 3dimensional space. Therefore, mass transport in the preferred direction takes place along a rough surface. Conditions for the potential energy dissipation are fulfilled when the height difference $\partial_{\|} h_{ \pm} \equiv h(i, j)-h\left(i+1, j_{ \pm}\right)-1$ along the direction of transport is positive. More precisely, since relaxation at a site involves two particles, the energy is dissipated at that site when the sum $\mathrm{E}(i, j) \equiv$ $\partial_{\|} h_{-}+\partial_{\|} h_{+}-1>0$.

Two comments are in order at this point: (1) Although the driving force of the grain transport in this model is not a gradient of the potential energy, as discussed in the Introduction, we believe that cumulative potential energy dissipated in an avalanche is an interesting quantity which is directly related to temporal fluctuations of the emergent structure in the real space. We concentrate on the properties of the energy distribution near the phase transition point $p=p^{\star}$, where the surface exhibits dramatic fluctuations, and only briefly discuss scaling behavior for $p>p^{\star}$. (2) We distinguish between energy dissipated at a fixed site, through which various avalanches run, and the energy dissipated at different points in the whole avalanche. Here we study scaling properties of the latter quantity. Notice that the condition $E(i, j)>0$ is fulfilled at a set of points $\mathcal{S}$ which is a random subset (see Fig. 1) of the avalanche size $s$ 14. Total energy dissipated in an avalanche is then $E=\sum_{\mathcal{S}} E(i, j)$.

The probability distribution of the dissipated energy $P(E)$ is found to obey a power-law behavior with the exponent $\tau_{E}$ for $p^{\star} \leq p<1$ and the following scaling form is satisfied

$$
P(E, L)=L^{\left(\tau_{E}-1\right) D_{E}} \mathcal{P}\left(E L^{-D_{E}}\right)
$$

with $\left(\tau_{E}-1\right) D_{E}=\tau_{t}-1$, where $\tau_{t}-1 \equiv \alpha$ is the survival probability distribution exponent. In Fig. 3 we show the integrated distribution of dissipated energies for $p=p^{\star}$ and for four different values of lattice size $L$. The slope gives the exponent $\tau_{E}-1=0.24$, and the finite-size scaling plot according to (4), which is shown in the inset to Fig. 3 , is obtained with $\alpha=0.45$ and $D_{E}=1.84$. In contrast to the survival probability distribution and size distribution of avalanches, the distribution of dissipated energy cannot be defined in the directed percolation processes, and thus the exponents $\tau_{E}$ and $D_{E}$ are new and are not directly related to the DP exponents. Moreover, we find that the exponents $\tau_{E}$ and $D_{E}$ are $p$-dependent, for instance, for $p=0.8$ we obtain $\tau_{E}=1.27$ and $D_{E}=$ 1.66 , and $\tau_{E}=1.29$ and $D_{E}=1.55$ for $p=0.9$. However, a combination of these exponents can be related to the survival probability exponent $\alpha$ via the scaling relation $\left(\tau_{E}-1\right) D_{E}=\alpha$, which holds in the SOC states, where $\alpha$ is the universal exponent expressible in terms of DP exponents via Eq. (2). This scaling relation is satisfied within numerical error bars (estimated as \pm 0.02 ) for all values of $p$ in the region $p^{\star} \leq p<1$. In the limit $p=1$ the critical state is exactly known [10] and consists only of the heights $h=1$ and $h=0$. Consequently, dissipated energy is bounded to values fife integer values, and the distribution $P(E)$ has the same scaling exponents as the size of avalanches distribution.

For $p^{\star} \leq p<1$ emergent spatial structure appears due to both stochastic dynamics and the conservation of number of particles in the interior of the pile [7]. At the edge of the scaling region $\left(p=p^{\star}\right)$ we find that the average height (averaged in the transverse direction) increases with the distance $\ell$ from the top row as $\left\langle h(\ell)>\approx a \ell^{b}\right.$, with $b=0.59 \pm 0.02$ (cf. Fig. 1 (top)). Therefore, for large $\ell$ there is a finite probability of large heights, however, at the same distance $\ell$ some site have height zero, since the system is in the stationary critical state (in the opposite the avalanche would propagate as a directed percolation cluster, which violates stationarity condition). Thus, $\partial_{\|} h$ also increases with $\ell$ and becomes unbounded for $\ell \rightarrow \infty$. Thus, the energy cutoff has additional nontrivial $\ell$-dependence, which is not contained in the $\ell$ dependence of the avalanche size cutoff, indicating that the sandpile surface is a fractal at $p=p^{\star}$. In the interior of the scaling region $\left(p^{\star}<p<1\right)$, the average height remains finite and not a function of $\ell$, however, height distribution does strongly depend on $p$. We find that the width of the height distribution $w(p)$ increases smoothly with decreasing $p$ from $w=1$ at $p=1$ to a fast diverging function at $p \rightarrow p^{\star}$. Neighboring sites are weakly correlated since the dynamics is governed by the critical height rule only, and thus dissipated energy at a site $E(i, j)$ also depends on $p$. We checked by direct calculation that the distribution of dissipated energy at a fixed site in the interior of the pile, $P\left(E_{\text {site }}\right)$, taken over $2 \times 10^{6}$ avalanches exhibits strong $p$-dependence. It is an exponential function of width $w_{e s}$, which is increasing smoothly with decreasing $p$ and becomes nearly powerlaw at $p=p^{\star}$. We believe that the $p$-dependence of the 
height distribution is the origin of the observed nonuniversality of the energy exponents. On the other hand, size and duration of avalanches are governed by the probabilities $p$ and $\rho^{\star}$, which sit always at the DK critical line in Fig. 2. It should be noticed that the probability $\rho(\ell)$ does not depend on particular values of heights $h>1$, and thus the avalanche exponents remain universal (cf. Eq. (2)).

We find that the distribution of mechanical work done by the system exhibits a curvature and not a power-law behavior.

\section{DYNAMIC PHASE TRANSITION}

In the region below $p^{\star}$ the system ceases to conduct and starts accumulating particles. As a consequence the critical steady state is lost (see detailed discussion in Ref. [7]) and the probability distributions exhibit exponential cut-offs with finite correlation length, depending on the distance from $p^{\star}$. In Fig. 4 we show the survival probability distribution $P(T, p)$ for few values of $p<p^{\star}$ and $L=200$. In general, a distribution $P(X, p, L)$ satisfies the following scaling form in the subcritical region

$$
P(X, p, L)=(\delta p)^{D_{X} \nu_{\|} \tau_{X}} \mathcal{P}\left(X(\delta p)^{D_{X} \nu_{\|}}, X L^{-D_{X}}\right),
$$

where $\delta p \equiv\left(p^{\star}-p\right) / p^{\star}$ and $X$ stand for $T, s$, or $E$, respectively, and $D_{X}$ is the corresponding fractal dimension. In the case of distribution of durations $P(T, p, L)$ we have $D_{T} \equiv z$ the dynamic exponent, and $z=1$ in the present model. Therefore there are no finite-size effects in the survival probability distribution, which makes it particularly suitable for the subcritical scaling analysis. In the case of size and energy distributions, one is restricted to values of $p$ and $L$ such that the condition $(\delta p)^{-\nu_{\|}} / L \ll 1$ is satisfied. In the inset to Fig. 4 the scaling collapse according to Eq. (5) of the survival probability distribution is shown, where we have used $\alpha=0.45$ and $z \nu_{\|}=1.22$.

Another way to study the dynamic phase transition is by direct measurements of the order parameter, i.e., the time-averaged outflow current $\langle J(p)\rangle$. In the critical steady state $\langle J(p)\rangle=1$, thus balancing the average input current. Below the transition point this balance is lost. The outflow current decreases reaching zero at some lower value of $p$, which depends on the system size $L$ [see Fig. 5 (bottom)]. For different lattice sizes we expect the following scaling form to hold:

$$
<J(p, L)>=L^{-\beta / \nu_{\|}} \mathcal{J}\left(L^{1 / \nu_{\|}}\left(p-p^{\star}\right) / p^{\star}\right) .
$$

This scaling form follows from general scaling properties of the internal current for $T<L$, that reads: $<j(T, p, L)>\sim L^{\lambda_{J}} j\left(L^{1 / \nu_{\|}}\left(p-p^{\star}\right) / p^{\star}, L^{-z} T\right)$. Ву choosing $L \sim\left(p / p^{\star}-1\right)^{-\nu_{\|}} \equiv \xi$ and having defined the exponent $\beta$ in Eq. (3), we find that the anomalous dimension $\lambda_{J}=-\beta / \nu_{\|}$. Therefore $\langle j(T, p, L)\rangle=$
$L^{-\beta / \nu_{\|}} \mathcal{G}\left(L^{1 / \nu_{\|}}\left(p-p^{\star}\right) / p^{\star}, L^{-z} T\right)$. In the stationary state for $p \geq p^{\star}$, correlation length $\xi \rightarrow \infty$ and the first argument in $\mathcal{G}$ can be neglected. For $T \ll L^{z}$ we expect that the scaling function $\mathcal{G}$ behaves as a power, i.e., $<j(T, p, L)>\sim$ const $\times T^{-\alpha} L^{z \alpha-\beta / \nu_{\|}}$, which should be independent on $L$, thus leading to $\beta / \nu_{\|}=z \alpha$. For the outflow current, however, we have $T \geq L$ and second argument of $\mathcal{G}$ can be neglected. Then for $p<p^{\star}$ one gets the expression (6). Taking $L^{1 / \nu_{\|}} \sim\left(p-p^{\star}\right) / p^{\star}$ leads to Eq. (3).

The scaling plot according to Eq. (6) is shown in Fig. 5 (top) where we have $\beta / \nu_{\|}=0.45$ and $1 / \nu_{\|}=0.83$. Together with the above results and observing the error bars for $\alpha$ from [7], we estimate the exponents as $\nu_{\|}=1.22 \pm 0.02$ and $\beta=0.56 \pm 0.02$.

Notice that the above values of the exponents $\nu_{\|}$and $\beta$ are close to the values $1.28 \pm 0.06$ and $0.58 \pm 0.06$, respectively, obtained by Monte Carlo simulations for $d=3$ dimensional directed percolation in Ref. 15. The reason for this similarity lies in the altered character of the dynamics of our model below the transition. Namely, the probability $\rho^{\star}$ of having height $h \geq 1$ reaches unity (in the limit of large $L$ ) at $p^{\star}$. The consequences of this are twofold: ( $i$ ) the threshold character of the dynamics is lost for $p<p^{\star}$, i.e., each site in the lattice satisfies the threshold condition $h \geq h_{c}=2$ when a single particle drops on that site; $(i i)$ since $\rho=1$ we have $P 1=P 2=p$, thus the system spreads the perturbation in the $\left(R_{\|}, R_{\perp}\right)$ plane with the probability $p$ and builds up heights with probability $q=1-p$. Therefore, for $0.5<p<p^{\star}$ we have a dynamic model in which an avalanche propagates effectively as a cluster in a 3-dimensional directed percolation with finite widths $\xi_{\|}, \xi_{\perp}$ in the plane, and percolating in the vertical direction. However, there are considerable differences between these processes and conventional 3dimensional DP, leading to generally different set of exponents, as discussed below. An added particle moves along rough surface $h\left(R_{\|}, R_{\perp}\right)$, which fluctuates inside the correlated region $\left(\xi_{\|}, \xi_{\perp}\right)$. The internal time scale becomes bounded by finite $\xi_{\|}$, and the system percolates for $t \rightarrow \infty$, where $t$ is now the external time scale (measured by the number of added particles). Flights of particles along the rough surface are proportional to local height gradients $\partial_{\|} h$, which are usually larger than one, in contrast to contact percolation processes. However, the average height of the pile $\langle h\rangle$ grows exactly by one unit with each added particle as a consequence of the conservation of number of particles.

\section{DISCUSSION AND CONCLUSIONS}

The dynamic model with stochastic relaxation rules of Eq. (1) exhibits the universal self-organized criticality for the range of toppling probabilities $p \leq p^{\star}<1$, 
and the dynamic phase transition at $p=p^{\star}$. As discussed in detail in Ref. [7], the avalanche exponents $\alpha$ for survival probability, $\tau \equiv \tau_{s}-1$ for integrated cluster size distribution, and $\zeta$ for the average transverse extent of clusters, are expressible in term of standard directed percolation exponents in all dimensions via Eq. (2). Here we have shown that the dissipated energy distribution, which is peculiar to the dynamic model and has no analogue in the directed percolation processes, is described by a new exponent $\tau_{E}$ and corresponding fractal dimension $D_{E}$. These exponents appear to be $p$ dependent, however, their product is related to the universal survival probability exponent due to scaling relation $\left(\tau_{E}-1\right) D_{E}=\alpha$. Power-law behavior of the distribution $D(E)$ at $p=p^{\star}$ indicates that the sandpile surface $h(i, j)$ is a fractal. We estimate the roughness exponent $\chi$ by measuring the time averaged height vs. transverse dimension of the pile $\left\langle h\left(R_{t r}\right)>\sim R_{t r}^{\chi}\right.$ at various distances $\ell$ from the first row [see Fig. 1 (top)]. By box counting we find that the contour curve of the perpendicular section through the pile for $p=p^{\star}$ has fractal dimension $d_{f}=1.44 \pm 0.045$, leading to $\chi=d_{f}-1=0.44 \pm 0.04$. Error bars are estimated from several separate measurements at different sections. The roughness exponent appears to be larger than the one measured in ricepile model with critical slope rules, where it was found $\chi_{R P}=0.23$ [3,16. In the steady state the height fluctuates around the average value $<h>=19 \pm 6$, increasing by one unit at boundary sites of an avalanche and decreasing by one unit at transport sites with one preceding active neighbor. The flame-like profile in Fig. 1 (top) indicates individual site fluctuations, in agreement with the critical height rules in our model. Closeness of the exponents $\chi \approx \alpha$ indicates that the number of sites at which the pile grows is on the average equal to the number of transport sites, i.e., the avalanches have almost no compact parts (cf. Fig. 1). Below the transition point the pile grows indefinitly for $t \rightarrow \infty$, as discussed in Sec. IV.

The dynamic phase transition at $p^{\star}$ is characterized by the exponents of the order parameter $\beta$, and the parallel correlation length $\nu_{\|}$, the numerical values of which appear to be very close to those of the directed percolation in $d+1$ dimensions. However, the exponent $\gamma$ for the order-parameter fluctuations and the exponent $\kappa$, which describes the average cluster growth at time $T$ as $m(T) \sim T^{\kappa}$, appear to be different from $d=3 \mathrm{DP}$ exponents (see Ref. [15). A complete set of exponents is given in Table I.

With regard to the exponents in Table I we would like to point out the following: (1) In the critical steady state the average cluster growth balances the average input of particles, i.e., $m(T) \sim 1$, leading to $\kappa=0$. The scaling relation $\kappa=\gamma / \nu_{\|}-1=D_{\|}-1-\alpha$ holds, thus we have $\gamma=\nu_{\|} ;(2)$ At the dynamic phase transition the hyperscaling relation (HS) $2 \beta+\gamma=[1+(d-1) \zeta] \nu_{\|}$appears to be violated 17,18, in contrast to the thermodynamic
DP phase transition (cf. Table I). The avalanche exponents are determined in Ref. [7]. The exponents for DP in $d=2$ are taken from Ref. 9 and the corresponding avalanche exponents $\tau_{D P}$ and $D_{D P}$ are calculated using the scaling relations. (Notice that due to the presence of anisotropy, at least three exponents should be known in order to determine completely the universality class.) The following scaling relations are valid both in DP and in dynamic models: $\beta / \nu_{\|}=\tau_{t}-1 \equiv \alpha$; $\beta /(\beta+\gamma)=\tau_{s}-1 \equiv \tau ; \zeta=\nu_{\perp} / \nu_{\|}$, and $D_{\|} \nu_{\|}=\beta+\gamma$. The hyperscaling violation exponent $\Omega$ is defined via $\beta+\gamma=\left[1+(d-1) \zeta-\beta / \nu_{\|}+\Omega\right] \nu_{\|}$, which together with the above equations leads to $\Omega=D_{\|}-1+\alpha-(d-1) \zeta$. Here $D_{\|}$is the fractal dimension of the size of relaxation clusters measured with respect to the length parallel to the transport direction. Using the fact that $D_{\|}=1+\alpha$ in the dynamic model, we may write $\Omega=2 \alpha-(d-1) \zeta$.

In the directed dynamic processes it is useful to define another exponent $\beta^{\prime}$, such that the generalized hyperscaling relation 18 20]

$$
\alpha\left(1+\beta^{\prime} / \beta\right)+\kappa=(d-1) \zeta,
$$

is satisfied. Here $\beta^{\prime}$ is related to the ultimate survival probability (the survival probability of a cluster grown from a fixed seed), whereas $\beta$ governs the usual orderparameter - stationary density of active sites. Recently exponential inequality $\beta^{\prime} \neq \beta$ was found in models with multiple absorbing configurations [19] and in branching annihilating random walks with even parity [20]. Our results in this paper suggest that in the self-organized dynamic critical states in directed models $\kappa \equiv 0$ and $\beta \neq \beta^{\prime}$ is always satisfied. We have

$$
\Omega=\frac{\beta}{\nu_{\|}}-\frac{\beta^{\prime}}{\nu_{\|}}>0 .
$$

Using the above scaling relations and Eq. (2) we find that $\beta^{\prime} / \nu_{\|}=\alpha_{D P}=0.159$ (cf. Table I). Therefore, the HS violation exponent $\Omega$ is given by the difference between the survival probability exponents in the dynamic model and the underlying directed percolation as $\Omega=\alpha-\alpha_{D P}$, which turns to be equal to the cluster growth exponent of DP, i.e., $\Omega=\kappa_{D P}$. One can also define a new configuration exponent $\gamma^{\prime}$ via $\gamma^{\prime} / \nu_{\|} \equiv \gamma / \nu_{\|}+\Omega$, such that the relation $2 \beta^{\prime}+\gamma^{\prime}=(1+(d-1) \zeta) \nu_{\|}$is satisfied. By inserting the above expression for $\Omega$ into $\gamma^{\prime}$, we have $\gamma^{\prime} / \nu_{\|}=1+\kappa_{D P}=1.314$. Using value of $\nu_{\|}$from Table 1 we find $\gamma^{\prime}=1.586 \pm 0.026$, and $\beta^{\prime}=0.195 \pm 0.012$. The origin of the difference between $\alpha$ and $\alpha_{D P}$ lies in the dynamic conservation law, which leads to the emergent spatial structure and dependence of the branching probability $\rho(\ell)$ on distance $\ell$, as discussed in Ref. [7]. Our present results suggest that the dynamic conservation law is also responsible for the new universality class of the dynamic phase transition at the edge of the critical region. 


\section{ACKNOWLEDGMENTS}

I thank Deepak Dhar for fruitful discussions and suggestions which led to the results presented in Fig. 2. I also thank Maya Pazsuski for helpful comments and suggestions. This work was supported by the Ministry of Science and Technology of the Republic of Slovenia.

* $\quad$ Electronic address: Bosiljka.Tadic@ijs.si

[1] P. Bak, How Nature Works, Copernicus Springer-Verlag, New York, 1996, and references therein.

[2] Y.-C. Zhang, Phys. Rev. Lett. 63, 470 (1989); S.S. Manna, J. Phys. A 24, L363 (1992); S. Maslov and Y.C. Zhang, Physica A 223, 1 (1996); A. Ben-Hur and O. Biham, Phys. Rev. E 53, R1317 (1996).

[3] K. Christensen, A. Corral, V. Frette, J. Feder, and T. Jøssang, Phys. Rev. Lett. 77, 107 (1996).

[4] S. Lübeck, B. Tadić, and K.D. Usadel, Phys. Rev. E 53, 2182 (1996).

[5] B. Tadić, Phys. Rev. E 57, 4375 (1998).

[6] The origin of stochasticity in biological processes has been discussed recently by D. R. Nelson and N. M. Shnerb, e-print cond-mat/9708071.

[7] B. Tadić and D. Dhar, Phys. Rev. Lett. 79, 1519 (1997).

[8] I. Derenyi and T. Vicsek, Phys. Rev. Lett. 75, 374 (1995); F. Jülicher and J. Prost, Phys. Rev. Lett. 75, 2618 (1995); e-print cpnd-mat/9611204.

[9] I. Jensen, J. Phys. A 29, 7013 (1996), and references therein.

[10] D. Dhar and R. Ramaswamy, Phys. Rev. Lett. 63, 1659 (1989).

[11] E. Domany and W. Kinzel, Phys. Rev. Lett. 53, 311 (1984).

[12] For $d=2$ the exponents of directed percolation are known very accurately, see Ref. [9].

[13] Notice that $\left\langle\rho^{\star}\right\rangle$ can be viewed as a response function of the system to a slowly increasing external "field" by one unit (particle) per time step.

[14] Set of red sites is a fractal with the same scaling properties as avalanche size.

[15] P. Grassberger, J. Phys. A 22, 3673 (1993).

[16] M. Paczuski and S. Boettcher, Phys. Rev. Lett. 77, 111 (1996).

[17] Hyperscaling violation has been discussed in relation to the phase transition in spin systems with quenched random fields. It implies an additional exponent $\tilde{\gamma}$, which governs the disconnected susceptibility, is introduced in order to determine completely the universality class of the transition [accurate values of the exponents are determined in Ref. M.E.J. Newman and G.T. Barkema, Phys. Rev. E 53, 393 (1996). See also discussion in Th. Jolicoeur and J.C. Le Guillou, e-print cond-mat/9706254.

[18] Violation of hyperscaling in DK cellular automaton was recently studied by R. Dickman and A. Yu. Tretyakov, e-print cond-mat/9504050.
[19] J.F.F. Mendez and R. Dickman, J. Phys. A 27, 3018 (1994).

[20] K.B. Lauritsen, P. Fröjdh, and M. Howard, e-prnt condmat/9808335.

TABLE I. Critical exponents in stochastic directed model $(\mathrm{PH})$ and in directed percolation (DP) in $d=2$ dimensions. Also shown are exact exponents in $p=1$ limit (DR).

\begin{tabular}{c||c|c|c|c|c||c|c|c|c|}
\hline \hline $\mathrm{M}-\mathrm{E}$ & $\alpha$ & $\tau$ & $D_{\|}$ & $\zeta$ & $\kappa$ & $\beta$ & $\nu_{\|}$ & $\gamma$ & $\Omega$ \\
\hline \hline $\mathrm{PH}$ & 0.46 & 0.31 & 1.46 & 0.62 & 0 & 0.56 & 1.22 & 1.22 & 0.30 \\
\hline $\mathrm{DR}$ & $1 / 2$ & $1 / 3$ & $3 / 2$ & $1 / 2$ & 0 & - & - & - & $1 / 2$ \\
\hline $\mathrm{DP}$ & 0.159 & 0.108 & 1.472 & 0.634 & 0.314 & 0.276 & 1.734 & 2.278 & 0 \\
\hline \hline
\end{tabular}
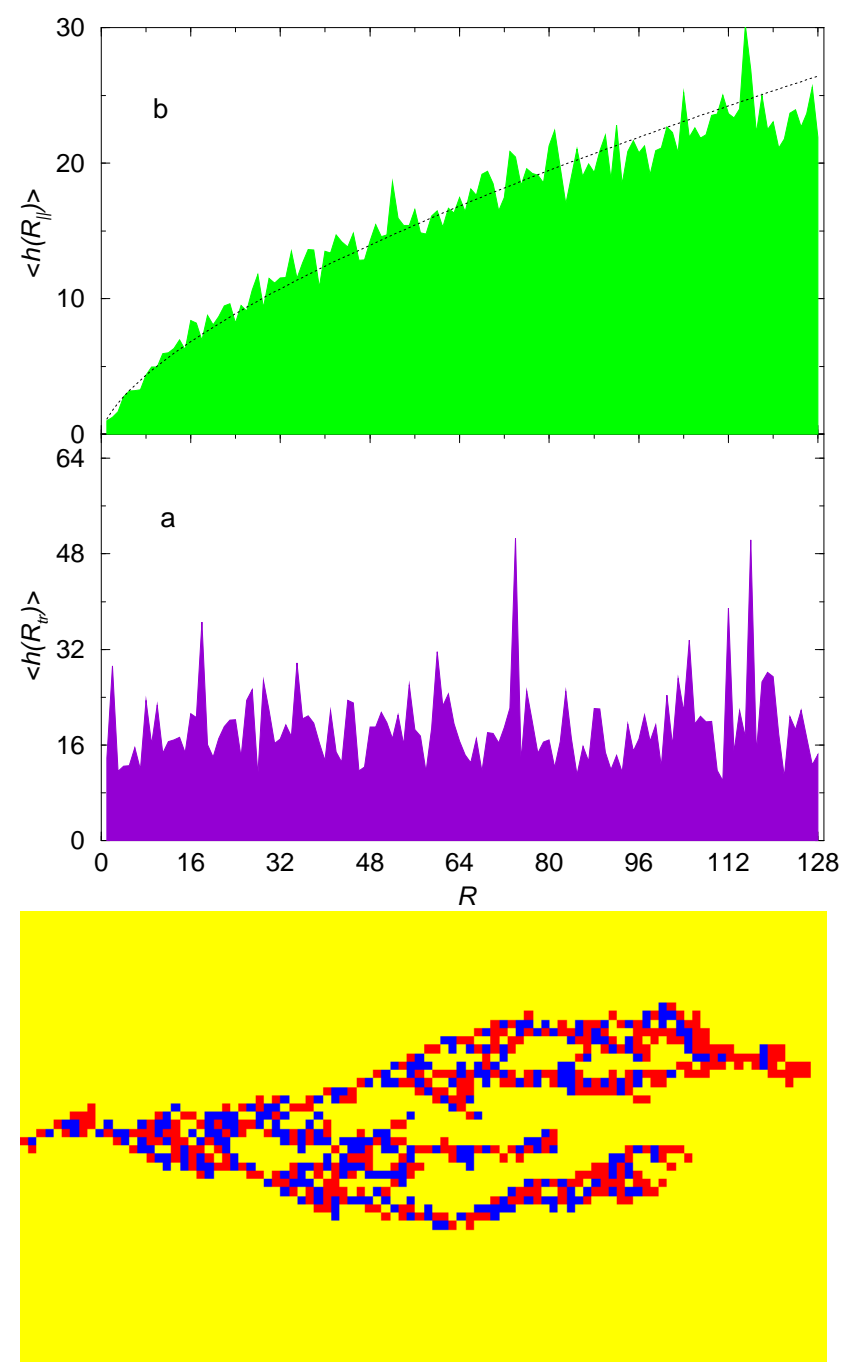

FIG. 1. Bottom: Avalanche in $1+1$ stochastic model for $p=p^{\star}$ and $L=128$. Transport direction is from left to right. Dark points represent sites at which system performs work, and at light dark points it dissipates energy. Top: (a) Transverse section of the pile at distance $\ell=96$ from the top. Heights are averaged over $5 \times 10^{6}$ runs. (b) Height (averaged in transferse direction) vs. parallel distance from the top in the stationary state. Dotted line: $1.6 \ell^{0.59}$. Heights are measured in number of grains and $R$ is given in lattice sites. 


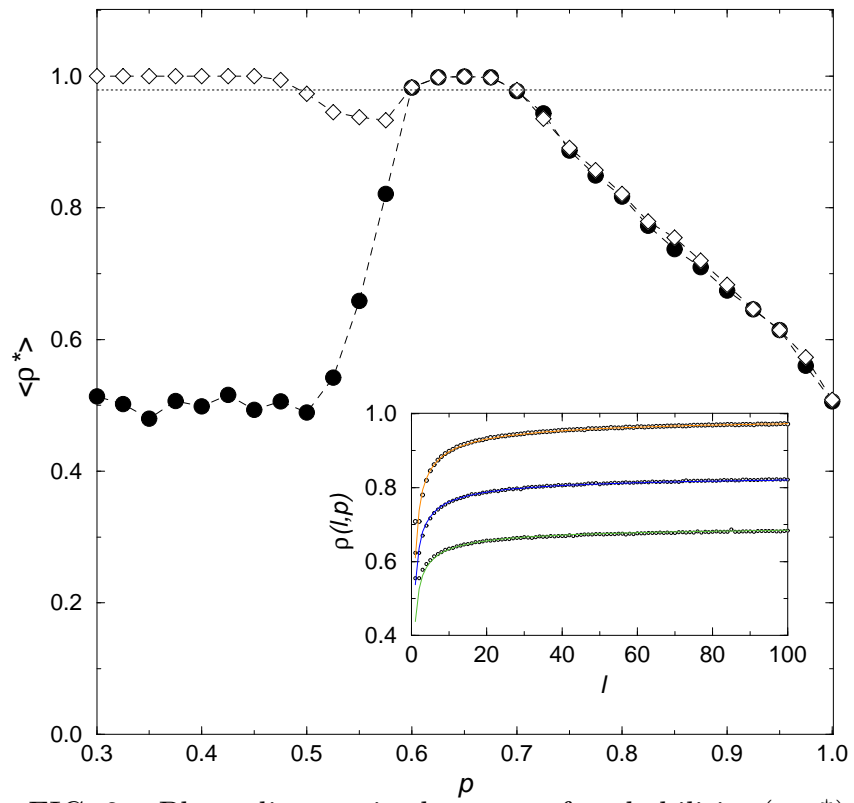

FIG. 2. Phase diagram in the space of probabilities $\left(p, \rho^{\star}\right)$ obtained numerically for a lattice with $L=100$. Open symbols correspond to initial conditions along the line $(p, 1)$, and filled symbols to initial conditions along the line $(p, 1 / 2)$. Dotted line indicates value $\left\langle\rho^{\star}>\right.$ at $p^{\star}$. Inset: $\rho(\ell)$ vs. $\ell$ for (top to bottom) $p=p^{\star}, 0.8$, and 0.9 , with fit lines described in the text.

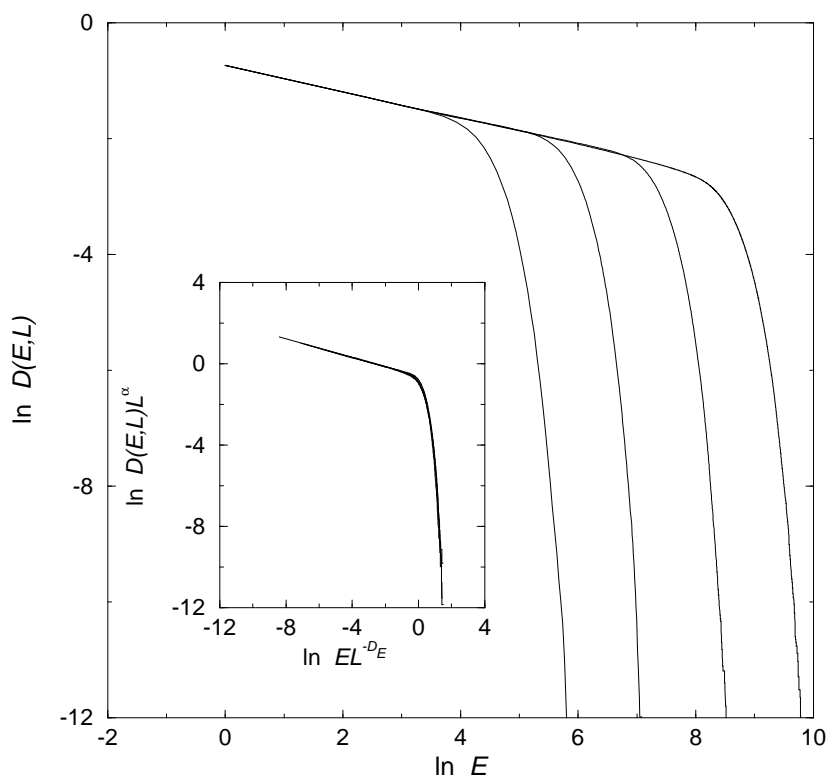

FIG. 3. Double logarithmic plot of the integrated distribution of dissipated energies $D(E, L)$ vs. $E$ for $p=p^{\star}$ and for $L=12,24,48$, and 96 (left to right). Inset: Scaling plot according to Eq. (4) with $\alpha=0.45$ and $D_{E}=1.84$.

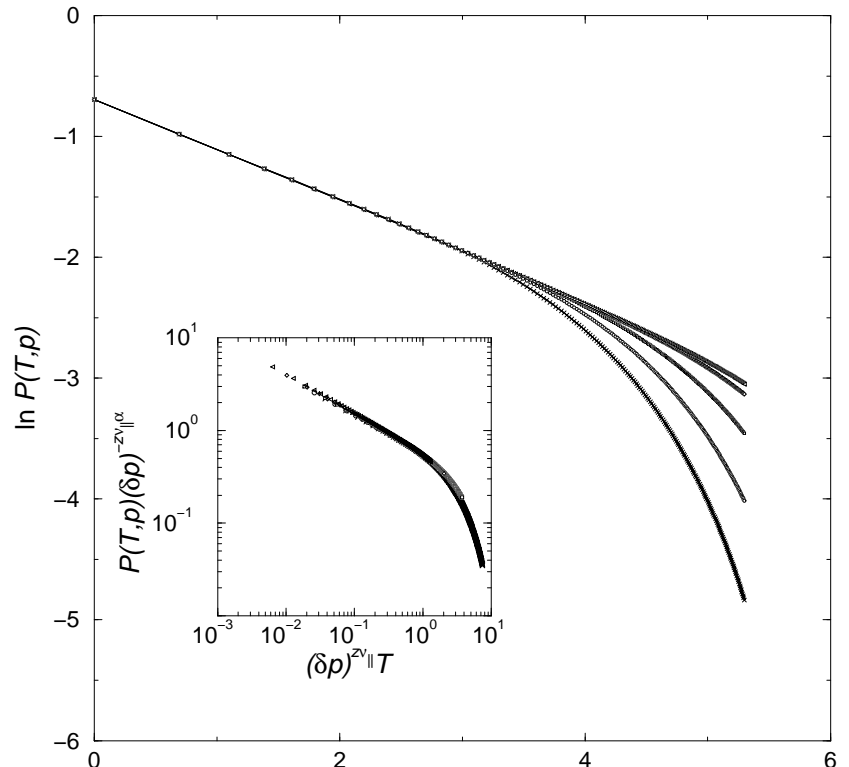

FIG. 4. Double logarithmi §lot of the integrated distribution of durations $P(T, p)$ vs. $T$ for $L=200$, and $p=0.695,0.69$, $0.68,0.67$, and 0.66 (top to bottom) in the subcritical region. Inset: Scaling plot according to Eq. (5) with $\delta p \equiv\left(p^{\star}-p\right) / p^{\star}$ and exponents $\alpha=0.45$ and $z \nu_{\|}=1.22$.
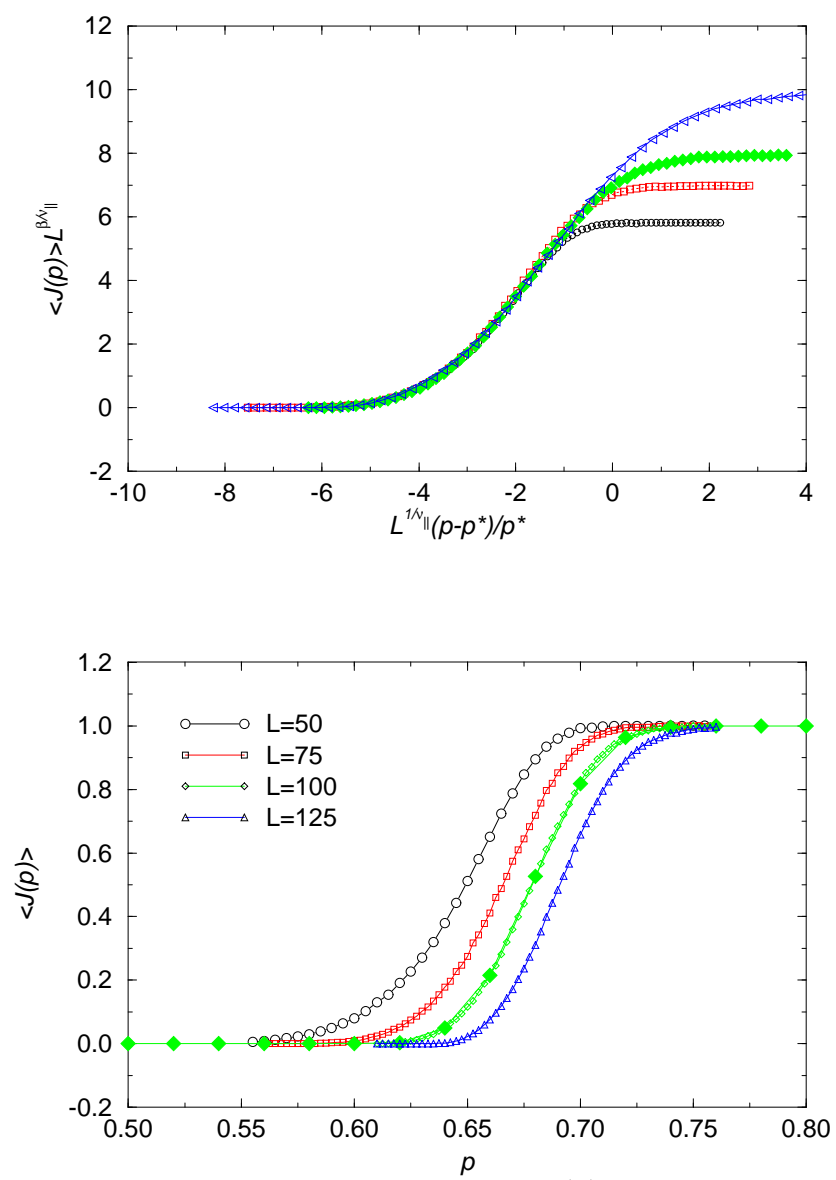

FIG. 5. Average outflow current $\langle J(p)\rangle$ vs. $p$ for various lattice sizes (bottom). Finite size scaling plot according to Eq. (6) with $\beta / \nu_{\|}=0.45$ and $1 / \nu_{\|}=0.83$ (top). 